\title{
Alfabetizando al alfabetizador: Mujeres hispanas en un nuevo contexto social
}

\section{Educating the Educator: Hispanic women in a new social context}

Investigación

Luis Javier Pentón Herrera

Doctorando Concordia University Chicago - USA luis.penton@gmail.com

Ángel A. Toledo López

Sistema Universitario Ana G. Méndez, Universidad del Este - Puerto Rico atoledo@suagm.edu

Recibido: 7 de noviembre de 2016 / Aceptado: 13 de diciembre de 2016

\section{Resumen}

La población hispana en Estados Unidos continúa aumentando establemente y de forma rápida. Este incremento fértil y constante de hispanos ha reorganizado el matiz social del país y expone a los inmigrantes a unas realidades sociales, lingüísticas y educativas no conocidas anteriormente. Estudiantes inmigrantes hispanos aprendices del idioma inglés son inmersos en un sistema educativo completamente desconocido y se enfrentan a un reto lingüístico que necesitan rebasar de forma diligente para tener éxito en sus escuelas. A su vez, los padres inmigrantes atraviesan otros retos similares en sus ambientes laborales en los cuales el idioma y otros componentes sociales les dificultan el éxito profesional y el involucramiento en las escuelas de sus hijos. Este artículo expone las realidades de los estudiantes hispanos y las madres hispanas en Estados Unidos a través de los ojos de cuatro madres hispanas inmigrantes. Mediante la entrevista profunda, este trabajo expone el impacto que tiene la participación parental en el desenvolvimiento escolar y académico de los estudiantes hispanos en Estados Unidos.

Descriptores: ELL, HSS, BLL, consorcio parental, inmigrantes hispanas, estudiantes hispanos.

\section{Abstract}

The Hispanic population in the United States continues growing steadily and rapidly. This fertile and constant growth has reorganized the country's social milieu and exposes immigrants to social, linguistic and educational realities that were previously unknown. Hispanic immigrant English Language Learners are immersed in a completely unfamiliar academic system and face linguistic challenges that must be overcome if they are to be successful in school. In the same way, immigrant parents undergo similar challenges in their work environments in which language and other social barriers impede their professional success and involvement in their children's academic life. This work presents the experiences of Hispanic students and mothers in the United States through the eyes of four Hispanic immigrant women. In depth interviews were used to test for the impact that parental involvement has in the academic development of Hispanic students in the United States.

Keywords: ELL, HSS, BLL, parental involvement, immigrant women, hispanic students.

Forma sugerida de citar: Pentón Herrera, Luis \& Toledo López, Ángel (2017). Alfabetizando al alfabetizador: Mujeres hispanas en un nuevo contexto social. Alteridad, 12(1), pp. 68-78. 


\section{Introducción}

La población hispana se duplicará en cantidad para el año 2060 (Buró del Censo de Estados Unidos, 2014). Es alarmante que el sistema educativo estadounidense no brinda a muchos estudiantes hispanos las herramientas necesarias para aprender inglés (Schneider, Martinez, y Owens, 2006), hacerse biletrados (Pentón Herrera y Duany, 2016) o graduarse (Balfanz et al., 2014). Esto provocará que, para el año 2060, se cree en Estados Unidos una brecha social y educativa aún mayor que dividirá generaciones de familias hispanas iletradas cuyos abuelos, padres e hijos no recibieron la educación necesaria para ser exitosos en sus vidas profesionales (Putman, et al., 2016). Contribuir al desarrollo de las futuras generaciones de estudiantes hispanos en Estados Unidos cobra, entonces, gran importancia.

La población actual de estudiantes hispanos que asiste a las escuelas primarias y secundarias en Estados Unidos se divide en dos grupos principales: (1) estudiantes inmigrantes hispanos aprendices del idioma inglés (ELLs por sus siglas en inglés) y (2) estudiantes hispanos que nacieron y crecieron en Estados Unidos, conocidos como hispanoparlantes por herencia (HSS por sus siglas en inglés). A pesar de que existe una gran diferencia en las realidades sociales y académicas de los ELLs y HSS, estudios demuestran que ambos grupos de estudiantes hispanos tienen un bajo rendimiento académico (Jacobs, 2016; Sparks, 2015). Por su parte, los HSS usan el español limitadamente. Esto, unido a la fuerte influencia que tiene el idioma inglés sobre sus vidas, tiene un impacto en el desarrollo de destrezas de alfabetización académica en ambos idiomas (Viola y Stefan, 2014).

Estudiantes inmigrantes de México, Guatemala, Honduras y El Salvador son actualmente la población de estudiantes ELLs de mayor crecimiento en las escuelas primarias y secundarias en Estados Unidos (Ferris y Raley, 2016). Una reciente publicación de Pentón Herrera y Duany (2016) destaca las necesidades académicas y lingüísticas de esta población estudiantil e introduce el concepto aprendiz binario de lenguajes (binate language learners o BLL por sus siglas en inglés) para describir a una gran proporción de estudiantes inmigrantes de estos países. Los BLLs son estudiantes que, además de ser ELLs, son iletrados, o tienen bajo aprovechamiento en sus destrezas lingüísticas en su lengua materna (L1), en este caso, español. Los BLLs llegan a las aulas estadounidenses con grandes deficiencias en destrezas lingüísticas en español que no fueron satisfechas en sus países natales. El problema es que las escuelas en Estados Unidos no tienen los recursos necesarios para llenar estas brechas. Como consecuencia, los padres se convierten en la fuente de apoyo más poderosa y en el puente que contribuye a crear redes de apoyo entre sus hijos, la escuela, la comunidad y el hogar. Estas redes de apoyo brindan la fortaleza necesaria para que los estudiantes se integren al proceso académico, tomen control de su proceso educativo y salgan airosos en el desarrollo de destrezas académicas y de lenguaje.

Muchos estudios sugieren que la participación de los padres en los asuntos académicos de sus hijos menores es clave en el éxito de estos últimos. Esto es particularmente cierto en el caso de estudiantes hispanos que asisten a escuelas en Estados Unidos (González et al., 2014; Hugo López, 2009; LaBahn, 1995; Panferov, 2010). Por el otro lado, otros estudios demuestran el impacto negativo que tiene la falta de participación parental en nuestra sociedad, nuestras escuelas y el éxito académico de estudiantes hispanos (LaBahn, 1995). Sin embargo, no existen muchos estudios que discutan las diferencias culturales y definan específicamente qué significa la participación parental.

Este estudio se enmarca en teorías sociológicas sobre roles sociales y motivación para auscultar cómo las madres hispanas se convierten en vehículos catalizadores del éxito académico de sus hijos. El estudio evalúa las posturas de madres inmigrantes hispanas que participaron de entrevistas profundas para analizar su sentir acerca de la importancia de educarse como adul- 
tas. Llegadas a la adultez, estas mujeres tenían la opción de trabajar o no y estudiar o no con el fin de lograr sus propósitos. Algunas utilizaron su propia educación como vehículo para promover el éxito estudiantil de sus hijos. Otras, valoraron su rol de madres dentro del núcleo familiar y optaron no seguir sus metas académicas. El estudio proveyó una oportunidad particular para evaluar tres principios esenciales: cómo la educación para adultos promueve el éxito académico de los hijos; cómo la educación para adultos promueve la participación parental en las escuelas de sus hijos; y, qué motivaciones o situaciones viabilizan que las madres inmigrantes hispanas opten por educarse y busquen mejores oportunidades laborales al llegar a Estados Unidos.

\section{Fundamentación teórica}

El impacto del consorcio parental en el éxito académico de los estudiantes es un tema que ocupa a maestros, administradores y generadores de política pública. En el campo de la educación, la participación o consorcio parental se define como la interacción de los padres con las escuelas y con sus hijos para promover el éxito académico de estos últimos (Kim y Hill, 2015). La participación parental se extiende no sólo al ámbito escolar, sino también al hogar. Esto significa que el consorcio parental incluye el compromiso de los padres para proveer, constante y consistentemente, recursos necesarios para que sus hijos alcancen el éxito en el ambiente académico

Varias investigaciones discuten la relación que existe entre el consorcio parental y el éxito académico de los hijos (Goodall y Montgomery, 2014; Harris y Goodall, 2008; Kim y Hill, 2015; Wilder, 2014). Estudios destacan el compromiso laboral como uno de los impedimentos más importantes para lograr que los padres se integren al proceso educativo de sus hijos (Harris y Goodall, 2008). A este se le unen el desconocer el idioma y el analfabetismo que impide que los padres engranen en el proceso académico y se involucren más en las escuelas. Como consecuen- cia, se han diseñado muchos programas para proveer a los padres la oportunidad de involucrase activamente para promover el éxito académico de sus hijos (Harris y Goodall, 2008). Estos programas identifican a los padres como agentes del éxito académico de los menores y cuenta con ellos para garantizar que los estudiantes tengan una oportunidad equitativa y justa de lograr sus metas educativas y profesionales.

El compromiso que los padres asumen en el hogar establece los cimientos para la exitosa ejecutoria académica de los hijos. Wilder (2014) encontró que el involucramiento parental tiene un impacto fuerte y positivo en el desarrollo de hábitos de estudio en los menores y en su posterior éxito académico. Este estudio identificó una correlación existente entre el involucramiento parental en el hogar y el aprovechamiento académico de los hijos. Cuando los padres se involucran en la vida académica de sus hijos desde el hogar, los estudiantes obtienen mejores calificaciones y son más exitosos en la escuela. De igual modo, Harris y Goodall (2008) afirman que el involucramiento parental en el proceso de aprendizaje de los hijos provoca enormes diferencias en el aprovechamiento académico de estos últimos. Esto implica que los padres tienen que involucrarse activamente en establecer normas, y guiar y trabajar con sus hijos estudiantes en la casa. Este compromiso activo por parte de los padres tiene un efecto positivo en el aprendizaje subsiguiente $y$ en el éxito profesional de los menores (Harris y Goodall, 2008).

Cuando los padres se involucran en la escuela, se crea una sinergia padre, hogar y escuela. Goodall y Montgomery (2014) encontraron que el involucramiento parental es instrumental en el proceso de regeneración de ambientes escolares particularmente en escuelas en las que los niños no están ejecutando según esperado. Además, los padres, como coeducadores en las escuelas, contribuyen a mejorar la cultura escolar. El involucramiento parental es un reflejo del interés que tienen los padres en el éxito académico de sus hijos. Por tal razón, los padres que se 
involucran activamente, fortalecen las relaciones familia-escuela y promueven el éxito de los hijos desde el hogar. Tomando esto en consideración, es imperativo considerar qué motivaciones viabilizan que los padres inmigrantes hispanos desarrollen las destrezas necesarias para su mejoramiento y para brindar apoyo a sus hijos.

Este análisis obliga a revisitar el tema de la sociología de la educación e integrar el tema de la cultura y las normas sociales establecidas. Las normas sociales que imperan en los países latinoamericanos se trasladan a Estados Unidos con cada inmigrante y se ponen en práctica entre grupos culturalmente homogéneos. Indudablemente, la cultura que se experimenta en el país de origen, particularmente los países de habla hispana, es diferente a la que se vive en Estados Unidos. Esto implica que, en este proceso de adaptación, se fortalecerán las prácticas sociales y culturales que se traen consigo. Los inmigrantes buscarán identificarse con grupos similares a los suyos de manera que puedan integrarse más eficazmente y acelerar el proceso de adaptación. Ahora bien, mientras más el inmigrante busca asociarse con aquellos grupos con los que se identifica, menos se integra a la cultura del nuevo país y menos necesidad tiene de aprender el nuevo idioma.

Uno de los rasgos que trae consigo el inmigrante y que implementa en el país al que inmigra es el de los roles de género. Lewis (2003) afirma que la diferenciación entre masculinidad y feminidad depende de temas de identidad nacional, clase, raza, religión y etnicidad, entre otros factores. Desde esta perspectiva, la definición de género es socialmente construida a la luz de las diferencias percibidas entre los determinantes biológicos de la mujer y el hombre. Esto implica que los roles de género, o las expectativas sociales que se les imponen a cada persona según su género, se crean culturalmente y se asignan a la luz de la construcción social basada en tradiciones y costumbres. Entonces, para entender algunos comportamientos sociales que adoptan las personas en el ámbito educativo, profesional o familiar, es imperativo conocer las diferencias sociales que imperan entre la masculinidad y la feminidad (Toledo López, 2002). Las expectativas sociales que se les imponen a los hombres y a las mujeres impactan sus comportamientos y las decisiones que toman a lo largo de su vida. El proceso de socialización lleva a los individuos a entender que deben comportarse de cierta manera para llenar las expectativas sociales que le han sido impuestas por razón de su sexo.

Estos patrones de conducta se recrean y se reviven en el extranjero cuando la familia hispana emigra al exterior. La mujer, por su parte, permanece limitada a los confines de la comunidad hispana donde escogió vivir para facilitar su integración, mientras que el hombre rompe las fronteras del barrio o la comunidad, para exponerse a los retos sociales, lingüísticos y económicos con el fin de proveer para su familia. Los roles de género que vivían en sus países de origen se reconstruyen en el nuevo ambiente en el que la mujer permanece bajo el control del hombre -esposo, padre, hermano o hijo- y es más o menos tratada como menor de edad (Bullough, 1979). Según Dávila (2013), la relación hombre-mujer entre los hispanos es una de hombre dominante y mujer sumisa en la que la mujer se deja llevar por normas sociales que las construyen dependientes e incapaces de asumir grandes responsabilidades. Esto explica porqué la mujer hispana, al llegar a Estados Unidos, se encierra dentro de las fronteras de su comunidad y no se expone a aprender el idioma. Se trata de un mecanismo de protección que, eventualmente, tendrá impacto directo en su vida y en la de sus hijos. Foster (1994) atribuye esta costumbre muy hispana a tres factores importantes: la disciplina militar que ha imperado en muchos países de Centro y Sur América; la moral católica que predomina en las sociedades hispanas; y las creencias familiares tradicionales hispanas que hacen que la mujer se circunscriba a unos roles claramente establecidos.

Este fenómeno cultural trae implicaciones serias, particularmente para la mujer hispana, dentro del nuevo contexto social al que se enfrenta. La mujer inmigrante hispana no encuentra razones válidas para educarse ni lingüística ni 
profesionalmente ya que, dentro de los confines de su comunidad, otro idioma además del español resulta innecesario. Trabajar tampoco le es requerido ya que su esposo es quien suple. Sus necesidades se establecen dentro de su nuevo entorno para garantizar la protección que alegadamente necesitan. Dentro del ambiente resguardado y protegido, se comunican en su idioma y cumplen con las labores que sus costumbres y tradiciones les permiten. Mientras el hombre exista, ellas atienden el hogar no solo porque ahí están protegidas, sino también porque ese es su trabajo como mujer. La crianza de los niños les compete a ellas, lo que también representa un reto enorme porque no pueden integrarse al ambiente escolar de estos ni pueden asistirlos en su proceso de aprendizaje e integración social, cultural y lingüística.

La ausencia del hombre en el cuadro familiar y social de la inmigrante hispana provoca que sus estructuras y necesidades se redefinan. Sus necesidades se reorganizan y asumen un nuevo orden jerárquico. Según Maslow (1943), las necesidades humanas se organizan jerárquicamente desde aquellas que plantean la plena subsistencia hasta las que competen la autorrealización. Para alcanzar necesidades de mayor jerarquía, es imperativo satisfacer aquellas que se encuentran en la base y que garantizan la vida y otras necesidades humanas básicas. Alcanzar determinadas metas, afirma Maslow (1943), motiva a los seres humanos a progresar hacia nuevos horizontes y satisfacer otras necesidades. Por ejemplo, una vez que se tiene garantizada la subsistencia, el techo y la cobija, puede darse paso a otras necesidades como el mejoramiento personal, el servicio comunitario y la acción creativa. Esta teoría nos permite comprender porqué, ante la presencia de una figura masculina en el hogar, la mujer hispana inmigrante en Estados Unidos no tiene motivos para educarse, aprender el idioma inglés y buscar integrarse a la cultura general estadounidense. Dentro de los límites comunales y de su hogar se siente protegida. Este sentido de protección no es tal que le permita moverse más allá para satisfacer otras necesidades, sino suficiente para sentir que vive con lo mínimo necesario. Se dedica a su familia y cumple con su rol de género, según su cultura lo establece. El reto comienza cuando el hombre -esposo, hijo, padre- se ausenta bien sea por muerte, mudanza, divorcio o cualquier otra razón. Esto reta los estándares sociales establecidos y obliga a la mujer inmigrante hispana a asumir posturas que antes no le eran requeridas. Esto rompe sus esquemas y les obliga a moverse de su zona de confort en un ambiente que, por mucho tiempo, le era desconocido. La motivación, entonces, surge a causa de un cambio en su situación de vida -la ausencia de la figura masculina- y la mueve a adelantar sus estudios y trabajar, lo que, a su vez, crea las condiciones para su integración en la vida académica de sus hijos. En fin, se presenta como una cadena de eventos que unidos, producen el enlace entre mejoramiento académico y profesional como adulta y el desempeño positivo de sus hijos en el ambiente escolar.

En resumen, este estudio plantea que las situaciones sociales y culturales de la mujer hispana inmigrante establecen el marco dentro del cual ella actúa al llegar a Estados Unidos. Mientras se perpetúan las circunstancias que le permiten mantener y revivir los roles de género que su cultura le impone, ellas se mantendrán ejecutando su rol de mujer cuidadora del hogar. Ahora bien, cuando se altera su entorno y cambian las situaciones, surgen las motivaciones necesarias para que ella rompa esquemas y busque satisfacer otras necesidades que no tenía como prioridad. $\mathrm{Al}$ buscar satisfacer otras necesidades, como las educativas y profesionales, se crean ambientes más propicios para que sus hijos menores también alcancen sus metas académicas en un ambiente saludable y estable. Con conocimiento del inglés, la mujer hispana inmigrante puede insertarse en el mercado del empleo y de la educación en Estados Unidos lo que, a su vez, le permitirá integrarse activamente en el proceso académico de sus hijos. De este modo, la educación adulta se establece como un mecanismo a través del cual se promueven los éxitos académicos de los menores. Gráficamente, se puede resumir esta hipótesis situacional/motivacional de la siguiente manera: 


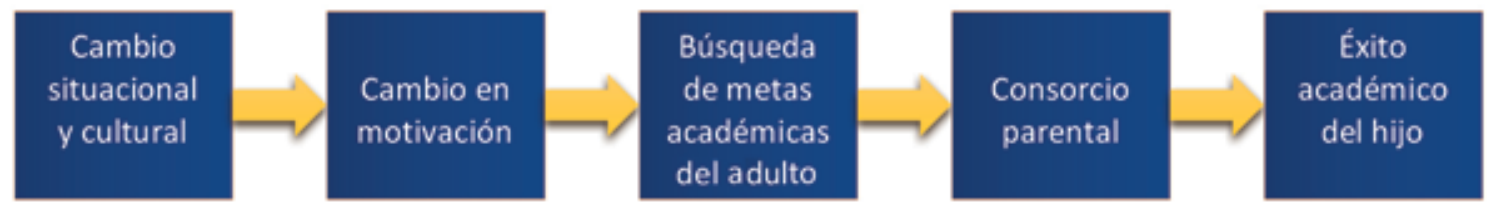

\section{Fundamentación metodológica}

Para corroborar esta hipótesis, se realizaron cuatro entrevistas profundas a cuatro mujeres hispanas que inmigraron a Estados Unidos. Se utilizó la variable de trabajo como factor de clasificación. Dicho de otro modo, sus características son esencialmente similares ya que todas son mujeres, hispanas, inmigrantes y madres, pero dos trabajan fuera del hogar y dos son amas de casa. Las entrevistas son en forma de estudios de caso sobre las experiencias de estas mujeres en el manejo de su entorno familiar. Los cuatro casos nos permiten analizar cómo el involucramiento, o falta de éste, de estas madres en los procesos académicos de sus hijos ha impactado el posterior desenvolvimiento de los jóvenes en el entorno escolar. De igual modo, las entrevistas arrojan luz sobre las motivaciones que tienen las madres participantes para buscar un trabajo, aprender el idioma inglés e involucrarse en los procesos académicos de sus hijos.

El estudio de caso resulta ser la metodología investigativa más apropiada para este análisis. Esta metodología permite explorar el fenómeno bajo estudio desde múltiples ópticas, lo que ayuda a ver el carácter multidimensional del tema (Baxter y Jack, 2008). Además, nos permite analizar el contexto dentro del cual ocurre el fenómeno bajo estudio (Yin, 2003). Ambas de estas fortalezas permiten que el estudio de caso se utilice con eficacia en el presente análisis. Este estudio mira porqué las mujeres hispanas inmigrantes optan por trabajar y aprender inglés como segundo idioma. Se enfoca en las condiciones contextuales de cada una de ellas y mira sus historias de vida para identificar factores que las motivaron o no a explorar alternativas laborales y lingüísti- cas. El análisis de estos casos permite ver cómo las diversas circunstancias impactan de diferentes formas a estas mujeres que participaron del estudio. Naturalmente, el análisis parte de unas variables control que permiten ver el impacto de las variables de estudio sobre las motivaciones y situaciones de las participantes. La decisión que toman estas mujeres de aprender o no un segundo idioma y de trabajar o no nos permite comprender las dinámicas de la educación para adultos y entender cómo la educación de las madres y su involucramiento en el proceso educativo de sus hijos impacta el desarrollo académico de los menores.

De modo que, las participantes de este estudio se ubican en uno de dos grupos: aquellas que trabajan y hablan inglés, y las que no trabajan y no hablan inglés. Provienen estas participantes de cuatro países de habla hispana, dígase, El Salvador, Cuba, Colombia y Honduras. Las preguntas efectuadas giraron en torno a la experiencia migratoria, el momento de entrada a Estados Unidos, el contexto familiar y social bajo el cual entraron y su conocimiento del idioma inglés al momento de entrar. De igual modo, se exploró su actual situación familiar, su conocimiento del inglés en el momento actual, la situación laboral y las motivaciones que tuvieron para cambiar su vida o permanecer bajo situaciones similares a las que existían cuando entraron a Estados Unidos.

\section{Análisis y resultados}

\section{Participantes bilingües que trabajan}

Dos participantes reportaron encontrarse trabajando al momento de la entrevista y capaces 
de comunicarse tanto en inglés como en español. Ambas llegaron a Estados Unidos hace más de dieciséis años, pero una comenzó a aprender inglés antes que la otra. La participante de Colombia, quien inmigró sola con sus hijas, comenzó su proceso de aprendizaje del segundo idioma tan pronto llegó a Estados Unidos. La participante de Honduras, sin embargo, mostró interés en aprender inglés a los nueve años de encontrarse en Estados Unidos. La participante colombiana indicó que el aprendizaje del inglés le resultó indispensable toda vez que tenía que proveer para sus hijas. Entendió que aprender inglés le permitía crecer profesionalmente de manera que pudiera ayudar a sus hijas a desenvolverse apropiadamente en un ambiente que les resultaba extraño. De igual modo, indicó que un factor de gran peso que la movió a aprender inglés de inmediato fue su necesidad de comprender el sistema educativo estadounidense y su funcionamiento. Ella valoró mucho el poder comunicarse con las maestras de sus hijas para identificar maneras en las que podía ayudar a las menores que pasaban por un proceso difícil de ajuste social, académico y lingüístico. Aprender inglés le permitió integrarse más fácilmente a su comunidad y emprender profesionalmente en un campo laboral de difícil manejo. De igual modo, le permitió comunicarse con las maestras de sus hijas, explorar alternativas de estudios para las menores y asistirles en su proceso académico. Le sirvió ella de modelo a ambas de sus hijas quienes hoy día son profesionales altamente capacitadas y con destrezas lingüísticas en ambos idiomas, inglés y español.

A su llegada, la participante colombiana descansaba en sus dos hijas para comunicarse. Las menores adquirieron destrezas en el idioma inglés con relativa facilidad. Se comunicaban son sus compañeros de escuela y con sus maestras, y demostraban tener éxito académico y buen ajuste social. En esa etapa, las dos menores asistían a su madre a ajustarse al nuevo contexto social y económico. Le servían de intérprete y le ayudaban a comunicarse efectivamente. Debido a que había llegado sola a Estados Unidos, la participante colombiana entendió imperativo aprender el idioma y trabajar de manera que pudiera proveer el sustento para sus hijas. Su motivación era clara y, en esencia, la situación a la que se enfrentaba la forzó a asumir roles importantes que, típicamente, nuestras sociedades asignaban a los hombres.

Por su parte, la participante hondureña, abandonó su país de origen en búsqueda de mejores condiciones de vida para su familia. Llegó a Estados Unidos con su entonces esposo y se acomodó en un ambiente social y cultural que le apoyaba sus raíces. Ante su desconocimiento del idioma y de las normas sociales de un nuevo país, la familia optó por buscar bolsillos culturales en los que la participante se sintiera cómoda. Desde ahí, podía cuidar a los hijos, conversar con personas de similar bagaje cultural y mantenerse en un ambiente resguardado y poco intimidante para ella. Su entonces esposo era quien se enfrentaba al diario fuera de la burbuja cultural y fue quien adquirió destrezas lingüísticas en inglés más fácilmente. Él se ocupaba de proveer para la familia y trabajar a diario, mientras que ella cuidaba de la familia y de la casa.

Su poca o ninguna exposición a contextos sociales estadounidenses retrasaron en la participante hondureña el proceso de adquisición de destrezas idiomáticas en inglés. Luego de la ruptura de su matrimonio, aprender inglés y trabajar dejaron de ser opciones. La responsabilidad parental de criar a sus hijos se complicó bastante no solo porque estaba sola, sino también porque no conocía el idioma. Indicó la participante hondureña que en Estados Unidos "quien no habla inglés, no es nadie". Ilustró su sentir explicando que asistir a sus hijos, proveerles apoyo académico, comunicarse con las maestras y socializar en los entornos de sus hijos le resultaba imposible. De igual modo, obtener un trabajo que le remunerara lo suficiente para mantener la familia a flote también era bastante cuesta arriba. Se vio obligada a aprender inglés por las circunstancias que le sobrevinieron luego de su separación. Tuvo que salir de su burbuja social y cultural 
para enfrentarse a un mundo desconocido en el que había vivido por espacio de nueve años. Finalmente, tuvo que visitar las escuelas de sus hijos para ponerse al día de los requisitos importantes, comunicarse con las maestras y ofrecer el apoyo que sus hijos necesitaban. Indica la participante hondureña que su principal motivación era ser un ejemplo para sus hijos. Posteriormente, se matriculó en un programa universitario para obtener una carrera y perfeccionar sus destrezas de comunicación escrita en inglés. Sus hijos, quienes ya se habían adaptado al sistema escolar estadounidense, vieron en el esfuerzo de ella un ejemplo de superación y de éxito. Hoy día continúan sus carreras escolares en búsqueda de un grado académico profesional.

\section{Participantes monolingües que no trabajan}

Dos participantes indicaron que actualmente no trabajan y que no se comunican en el idioma inglés. Ambas han vivido en Estados Unidos por más de diez años y ambas indican que el español es su idioma materno. Ambas indican que la razón primordial para no aventurarse a trabajar ni a aprender el inglés es que prefieren dedicarse a sus familias. Intentaron asistir a escuelas de idiomas para adquirir destrezas lingüísticas en inglés, pero entendieron que esto les quitaba tiempo para estar con sus familias. Irónicamente, la participante salvadoreña indicó que su desconocimiento del inglés le impide asistir a su hija en su proceso académico ahora que se encuentra en la escuela. Sin embargo, ambas admiten que aprender un segundo idioma promueve el crecimiento personal y profesional, y provee una herramienta esencial para alcanzar metas laborales.

Las dos participantes aclararon que se sienten muy incómodas de su incapacidad de comunicarse en inglés, pero que ya es muy poco lo que pueden hacer porque les corresponde atender a sus familias. La participante cubana indicó: "no me siento cómoda con mi conocimiento del lenguaje. Ahora, lo único que me restaría es quitarle tiempo a mi hijo para aprender inglés, y no voy a hacer eso". Para ambas participantes, resulta muy difícil manejarse en un ambiente extraño cuando no conocen el idioma. Se sienten inseguras y, como consecuencia, se han limitado a un ambiente muy cerrado en el que comparten solo con la comunidad y con las personas del hogar. Todavía no ven la necesidad de aprender el idioma, aun cuando esto les impida involucrarse más en las escuelas de sus hijos, establecer lazos con los maestros y contribuir al proceso académico y éxito escolar de los menores. Ambas dependen de sus esposos para moverse, comunicarse y socializar. Sus formas de sustento están supeditadas a la capacidad económica que puedan lograr los esposos y a lo que ellos puedan proveer. Muestran lamento por no poder ayudar más a sus hijos en sus faenas escolares porque ellas mismas no están preparadas académicamente ni conocen el inglés. Esta situación es particularmente más difícil para la participante salvadoreña quien reside en el estado de Maryland, Estados Unidos en donde imperan los sistemas escolares predominantemente anglosajones con algunos bolsillos sociales y culturales que corresponden a la comunidad hispana. El hijo de la participante cubana no muestra tanta dificultad académica como la hija de la participante salvadoreña porque este y su familia residen en Miami, Florida, donde la población y la cultura hispana está entronizada a través de los inmigrantes cubanos. Esto no implica, sin embargo, que el hijo de la participante cubana no muestra dificultades académicas en la escuela, sino que ella, debido a que algunas maestras de su hijo hablan español, puede comunicarse con ellas para conocer sobre los problemas que enfrenta su hijo. Lo que no puede hacer, porque no tiene las destrezas académicas ni lingüísticas suficientes, es apoyar al menor desde el hogar para solidificar las herramientas y el soporte que recibe en la escuela.

\section{Discusión y conclusiones}

La finalidad de este estudio es evaluar cómo los cambios situacionales en la vida de las mujeres inmigrantes hispanas pueden impactar su entor- 
no, romper sus esquemas y motivarlas a perseguir metas académicas y lingüísticas que redundarán en beneficios para ella y su familia. Más específicamente, el estudio busca identificar si la educación de adultos se convierte en vehículo para fomentar la excelencia académica en los hijos y propiciar ambientes de enlace entre el hogar y la escuela. La teoría establece que una mayor participación o consorcio parental con la escuela provoca mayor aprovechamiento académico en los hijos. Los padres que se involucran activamente en las actividades escolares de sus hijos tanto en el hogar como en la escuela, logran que los menores se adapten más rápidamente al ambiente escolar y que muestren mayor aprovechamiento académico que sus contrapartes. Ahora bien, para que se dé el consorcio parental, tienen que existir las condiciones que lo propicien entre aquellos llamados a ocuparse de los menores.

En las culturas hispanas, son las madres las que cargan con la responsabilidad de criar a sus hijos menores. Un enfoque en las teorías de asignación de roles de género permite ver que las sociedades hispanas, debido a las tradiciones entronizadas, la moral religiosa y la cultura militar, promueve que el hombre sea el jefe de la casa y proveedor, mientras que la mujer cuida de los menores y atiende las faenas diarias del hogar. Cuando emigran a Estados Unidos, estas familias traen consigo sus tradiciones culturales y las recrean en un ambiente social que les resulta cómodo y en el que la mujer está protegida. Es el hombre quien se enfrenta al ambiente desconocido, aprende el idioma y se integra lo más rápido posible al ambiente laboral. Esto implica, sin embargo, que la mujer queda rezagada y, quizás, recluida en los confines comunitarios que le resultan conocidos y familiares. El cambio situacional y, como consecuencia, esquemático-social, ocurre cuando desaparece la figura masculina del panorama familiar. Esto puede ocurrir por razón de divorcio, muerte, viaje o cualquier otra razón que separe al asignado proveedor del núcleo familiar al que alimenta y sostiene. Ante este nuevo panorama, la mujer inmigrante hispana se ve en la obligación de adoptar roles que le son extraños en un ambiente que, por su segregación, le resulta también extraño. Consecuencia de esto es que esta mujer ve en su educación adulta un vehículo para alcanzar metas que anteriormente no podía alcanzar. Valora, como resultado, el adaptarse al ambiente social más allá de la comunidad y aprender el idioma de la metrópolis para integrarse a un mundo laboral que le permitirá proveer el sustento para su familia.

Este estudio valida el argumento situacional/motivacional y su conexión con las consecuencias académicas para los estudiantes menores de edad dependientes de las madres participantes. En los hogares en los que se produjeron cambios en el ambiente familiar, las participantes se vieron motivadas a aprender el idioma inglés y obtener una carrera profesional. Ambos recursos, según ellas, se convirtieron en el vehículo para alcanzar mayor estabilidad económica y seguridad en el hogar. Mientras aprendían el idioma, se integraban al mundo laboral de manera que proveyeran para el sustento de sus menores. Ambas vieron que este cambio les permitió crear lazos importantísimos con los maestros de sus hijos que redundaron en beneficios para los menores. Estas madres pudieron dar apoyo académico desde el hogar. Esto facilitó la integración de los menores al ambiente académico que desconocían. De igual manera, las madres se hicieron más participativas en las escuelas de sus hijos, lo que les permitió dar más y mejor seguimiento al progreso académico de estos. Ambas afirmaron que esta integración les sirvió tanto a ellas como a los menores en sus respectivos procesos de integración social, cultural y académica.

Por su parte, las participantes que optaron por mantenerse en el hogar para cumplir con sus roles femeninos hoy día resienten el no haber aprendido inglés. Están felices porque pudieron darles a sus hijos calidad de vida familiar en el hogar, pero reconocen que no pudieron darles soporte académico que facilitara su integración a un ambiente escolar desconocido para ellos. Ambas afirmaron la importancia de aprender el 
idioma como adultas y de educarse independientemente de su edad, pero coincidieron en que se les había hecho demasiado tarde porque su prioridad seguía siendo su familia. Es importante señalar que en ambos de estos casos imperaba la estructura familiar tradicional en la que el hombre ostentaba la posición de cabeza de familia y proveedor de las necesidades del hogar.

Este análisis, preliminar de cierto modo, evidencia el vínculo que existe entre la educación para adultos y el éxito académico de los hijos. Cuando alfabetizamos a la alfabetizadora, le damos las herramientas que necesita para superarse y para viabilizar la superación y el éxito de quienes dependen de ella. La sinergia madre-hogar-escuela permite que los hijos se desarrollen en un ambiente protegido y resguardado que les dé la estabilidad que necesitan para integrarse lingüística y académicamente al ambiente escolar dentro del nuevo contexto. La madre (y el padre también), como modelo y ejemplo, en la medida en que se educa promueve el crecimiento propio y el de sus hijos. Esta madre (y padre) busca integrarse al ambiente escolar para traer la escuela a la casa y hacer al maestro partícipe de las experiencias de sus hijos. Esta combinación promueve el crecimiento y la excelencia académica tan necesaria en un mundo globalizado y competitivo como el de hoy.

\section{Referencias bibliográficas}

Balfanz, R., Bridgeland, J. M., Fox, J. H., DePaoli, J. L., Ingram, E. S., \& Maushard, M. (2014). Building a grad nation: Progress and challenge in ending the high school dropout epidemic. Civic Enterprises. Recuperado de http://files. eric.ed.gov/fulltext/ED556758.pdf

Bullough, V. (1979). Homosexuality: A history. New York, NY: A Meridian Book, New American Library.

Dávila, J. (2013). Sexualidad, género y sociedad en la literatura del Caribe hispanoparlante (19502000) (Doctoral dissertation). Recuperado de ProQuest LLC. (UMI Number 3561667).

Ferris, M. \& Raley, S. (2016). The elephant in the classroom: Mass immigration's impact on public education. Fair Horizon Press. Rescatado de http://www.fairus.org/DocServer/researchpub/FAIR_2016_Education_Report.pdf

Foster, D. (1994). Cultural diversity in Latin American literature. Albuquerque, NM: University of New Mexico Press.

Gonzalez, C., Hutchins, M. C., Hernandez, C., Llama, M., Rossie, M., \& Suarez, S. (2014).

Parents Acquiring Learning Strategies (PALS): How empowering Hispanic parents increases parental involvement and student achievement. Recuperado de http://citeseerx. ist.psu.edu/viewdoc/download?doi=10.1.1.4 $25.6722 \&$ rep $=$ rep $1 \&$ type $=$ pdf

Goodall, J. \& Montgomery, C. (2014). Parental involvement to parental engagement: A Continuum. Educational Review, 66(4), pp. 399-410.

Harris, A. \& Goodall, J. (2008). Do parents know they matter? Engaging all parents in learning. Educational Research, 50(3), pp. 277-289.

Lopez,M.H.(2009).Latinos and education: Explaining the attainment gap. Pew Research Center: Hispanic Trends. Recuperado de http://www. pewhispanic.org/2009/10/07/latinos-andeducation-explaining-the- attainment-gap/

Jacobs, J. (2016). Learning English: Accountability, common core and the college-for-all movement are transforming instruction. Education Next, 16(1), pp. 38-45.

Kim, W. S. \& Hill, N. E. (2015). Including fathers in the picture: A meta-analysis of parental involvement and students' academic achievement. Journal of Educational Psychology, 107(4), pp. 919-934.

LaBahn, J. (1995). Education and parental involvement in secondary schools: Problems, solutions and effects. Educational Psychology Interactive. Recuperado de http://www.edpsycinteractive.org/files/parinvol.html

Lewis, L. (2003). Introduction. En: L. Lewis (Ed.), The culture of gender and sexuality in the Caribbean (pp. 1-21). Gainesville, FL: University Press of Florida.

Maslow, A. (1943). A theory of human motivation. Psychological review, 50(4), 370-396.

Panferov, S. (2010). Increasing ELL parental involvement in our schools: Learning from the parents. Theory into Practice, 49(2), 106-112. 
Pentón Herrera, L. J. \& Duany, M. (2016). Native Spanish speakers as binate language learners. NECTFL Review, 78, pp. 15-30.

Putman, H., Hansen, M., Walsh, K., \& Quintero, D. (2016). High hopes and harsh realities: The real challenges to building a diverse workforce. Brown Center on Education Policy. Recuperado de https://www.brookings.edu/ wp- content/uploads/2016/08/browncenter_20160818_teacherdiversityreportpr_ hansen.pdf

Schneider, B. Martinez, S., \& Owens, A. (2006). Barriers to educational opportunities for Hispanics in the U.S. En: M. Tienda (Ed.), Hispanics and the future of America (pp. 179227). Washington, DC: National Academies Press.

Sparks, S. D. (2015). The building assets-reducing risks program: Replication and expansion of an effective strategy to turn around low- achieving schools. Education Week, 35(11), pp. 5.

Toledo López, A. (2002). Prejudice or proxy: Candidate gender, information, and the vote choice (Doctoral dissertation). Recuperado de ProQuest Information and Learning Company (UMI Number 3077014).

United States Census Bureau (2014). Population Estimates, National Total: Vintage 2014. Rescatado de http://www.census.gov/popest/ data/national/totals/2014/index.html

Viola, G. M. \& Stefan, T. G. (2014). Heritage speakers' Spanish in California: How unbalanced bilingualism affects reverse constructions of the gustar-type. Recuperado de http://citeseerx. ist.psu.edu/viewdoc/download?doi=10.1.1.4 32.7649\&rep $=$ rep $1 \&$ type $=$ pdf

Wilder, S. (2014). Effects of parental involvement on academic achievement: A meta-synthesis. Educational Review, 66(3), pp. 377-397. 